\title{
ADSORÇÃO DE FÓSFORO E SUA RELAÇÃO COM FORMAS DE FERRO EM DEZ SOLOS DO URUGUAI ${ }^{(1)}$
}

\author{
J. HERNÁNDEZ ${ }^{(2)} \&$ E. J . ME URER ${ }^{(3)}$
}

\begin{abstract}
RESUMO
Oxihidróxidos de ferro formam ligações químicas muito fortes com ânions fosfatos, diminuindo suas disponibilidades para as plantas. Este trabalho foi realizado em 1994 e 1995, com os objetivos de quantificar formas de ferro em solos do Uruguai e de relacioná-las com a adsorção de fósforo. Em amostras superficiais $(0 \mathrm{a} 15 \mathrm{~cm})$ do horizonte $A$ de dez solos, o ferro extraído com ditionito $\left(\mathrm{Fe}_{\mathrm{d}}\right)$ variou entre 1.598 e $8.592 \mathrm{mg} \mathrm{kg}^{-1}$ e esteve relacionado com o material de origem. As formas de ferro de baixa cristalinidade, extraídas com oxalato de amônio 0,2 mol L-1 pH $3\left(\mathrm{Fe}_{03}\right)$, representaram de 45 a 78\% do total extraído com o ditionito. A capacidade máxi ma de adsorção de fósforo $\left(K_{2}\right)$, calculada a partir do modelo de Langmuir, variou de 104 a $704 \mathrm{mg} \mathrm{kg}^{-1}$. Encontrou-se correlação significativa $(r=0,894, P<0,01)$ entre as formas de óxidos de ferro de baixa cristalini dade $\left(\mathrm{Fe}_{03}\right)$ ea adsorção de fósforo pelos solos. A porcentagem de fósforo adsorvida pelos solos após a adição de $600 \mathrm{mg}$ de $P$ kg $^{-1}\left(P_{600}\right)$ relacionou-se significati vamente $(r=0,975, P<0,01)$ com a capacidade máxima de adsorção de fósforo pelos solos. Também apresentou alta correlação $(r=0,894, P<0,01)$ com as formas de ferro de baixa cristalinidade $\left(\mathrm{Fe}_{03}\right)$, e pode ser utilizado como um índice para estimar a capacidade de adsorção de fósforo desses solos.
\end{abstract}

Termos de indexação: adsorção de fósforo, adsorção máxima, energia de ligação, equação de Langmuir, óxidos de ferro.

\section{SUMMARY: PHOSPHORUS SORPTION IN SOILS FROM URUGUAY AND ITS RELATIONSHIP WITH IRON OXIDES}

I ron oxihydroxides form strong chemical bonds with phosphate anions, reducing $\mathrm{P}$ availability to plants. The objective of this study, conducted in 1994/ 95, was to quantify

\footnotetext{
(1) Parte da Tese de Mestrado em Ciência do Solo do primeiro autor, apresentada à Universidade Federal do Rio Grande do Sul em agosto de 1996. Recebido para publicação em novembro de 1996 e aprovado em maio de 1998.

(2) Professor da Facultad de Agronomia. Universidad de la Republica. Montivideo, Uruguay.

(3) Professor do Departamento de Solos da Faculdade de Agronomia, Universidade Federal do Rio Grande do Sul. Caixa Postal 776, CEP 90001-970 Porto Alegre (RS). Bolsista do CNPq.
} 
forms of iron oxides in soil s from Uruguay, and to study their re ationships with phosphorus sorption. I ron oxides extracted with dithi onitecitrate-bicarbonate $\left(\mathrm{Fe}_{d}\right)$ from ten soil samples (0-15 cm depth) ranged from 1598 to $8.592 \mathrm{mg} \mathrm{kg}^{-1}$ and were related with their parent material. Poorly ordered Fe-oxihydroxides, extracted by $0.2 \mathrm{~mol} \mathrm{~L}^{-1}$ ammonium oxalate at $\mathrm{pH} 3\left(\mathrm{Fe}_{03}\right)$, ranged from 45.2 to $78.2 \%$ of the total iron oxides extracted by dithionite The maximum P-sorption soil capacity $\left(\mathrm{K}_{2}\right)$, estimated by the Langmuir equation, ranged from 104 to $704 \mathrm{mg} \mathrm{kg}^{-1}$ of soil. The P sorption was strongly correl ated $(r=0.894, \mathrm{P}<0.01)$ with the poorly ordered iron oxides $\left(\mathrm{Fe}_{03}\right)$. The percent of phosphorus sorbed by soils after equilibrium with $600 \mathrm{mg} \mathrm{kg}^{-1} \mathrm{P}\left(\mathrm{P}_{600}\right)$, was significantly correlated $(r=0.975, \mathrm{P}<0.01)$ with the maximum P-sorption capacity and, with poorly crystalline Feminerals ( $r=0.894$, $\mathrm{P}<0.01$ ) extracted by the $0.2 \mathrm{~mol} \mathrm{~L}^{-1}$ oxalate ammonium $\mathrm{pH}$ 3. This index can be used to estimatethe P-sorption capacity of these soils.

Index terms: phosphorus sorption, maximum adsorption, binding energy, Langmuir equation, iron oxides.

\section{INTRODUÇÃO}

Os sol os do U ruguai são natural mente deficientes em fósforo, razão porque é necessária sua aplicação através de fertilizantes, para evitar limitações nutricionais para as culturas e pastagens. Entretanto, têm sido observadas diferenças entre solos, na eficiência de utilização de fósforo pelas plantas, relacionadas com processos deadsorção. Um dos fatores relativos à adsorção de fósforo em solos é o conteúdo de óxidos de ferro. Os solos do país apresentam, em geral, baixos teores totais de óxidos de ferro e proporções variáveis em formas de baixa cristalinidade (Cayssials \& Puentes, 1974; Hernández et al., 1985). As formas de ferro de baixa cristalinidade apresentam maior área superficial específica, o que determina maior reatividade química com os fosfatos (Bromfield, 1965; Syers et al., 1971; Ballard \& Fiskell, 1974; Madrid \& Arambarri, 1985; Dick, 1986).

As variações no regime hídrico dos solos ocasionam variações temporais nas condições de oxi dação-redução dos sol os. Tais variações, associadas com altos teores naturais de matéria orgânica, podem determinar condições favoráveis à formação de compostos de ferro de baixa cristalinidade (Kämpf, 1988).

Diferentes métodos são utilizados para identificar as formas de ferro em solos. O método de redução pelo ditionito de sódio é o mais utilizado, para caracterizar a fração de óxi dos de ferrolivres (Mehra \&J ackson, 1960). Essetratamento dá como resultado a dissolução completa dos óxidos de ferro, sem diferenciar as distintas formas ou compostos que podem estar relacionados com os processos de adsorção do fósforo. As frações dos óxidos de ferro extraídas dos solos pelo oxalato de amônio a pH 3 têm-se relacionado com a adsorção dofósforo (K halid et al., 1977; Willett et al., 1978; Willett \& Higgins,
1978, 1980; Bradley et al., 1984). Sah et al. (1989) constataram que a fração de ferro extraída com oxalato de amônio a pH 6, antes da extração convencional a pH 3, apresentou melhor correlação com o fósforo adsorvido por sol os sujeitos a períodos alternados de alagamento e drenagem. Esse método foi também utilizado por Vahl et al. (1993), para estimar, em amostras secas, o conteúdo de ferro que foi transformado ao estado reduzido após alagamento do solo para a cultura de arroz.

Os objetivos deste trabal ho foram: a) quantificar os teores eformas de óxidos de ferro em dez solos do Uruguai, sujeitos a variações temporais nas condições de oxidação-redução, e b) relacionar esses teores e formas de ferro com a adsorção de fósforo.

\section{MATERIAL E MÉTODOS}

Foram coletadas amostras dos horizontes superficiais (0 a $15 \mathrm{~cm}$ ) de dez solos do Uruguai, de importância na produção de pastagens e/ou sistemas mistos arroz-pastagens. A característica comum dos solos estudados é a de ocupar posições baixas da paisagem, pelo que estão sujeitos à alternância de períodos de excesso de água com períodos de seca, o que determina mudanças nas condições de oxidaçãoredução de duração variável. As amostras foram secas ao ar, moídas e peneiradas (malhas de $2 \mathrm{~mm}$ de diâmetro). Nos quadros 1 e 2, apresentam-se características selecionadas dos sol os estudados.

\section{Caracterização das frações de ferro}

Foram usados os seguintes métodos e extratores: a) ferro total extraído pelo ditionito de sódio $\left(\mathrm{Fe}_{\mathrm{d}}\right)$, avaliado pela técnica proposta por Kilmer (1960), modificada edescrita em Cayssials \& Puentes (1974); duplicatas de 1,25 g de sol oforam colocadas em tubos 
Quadro 1. Classificação, material de origem e teor de fósforo dos dez solos estudados (camada de 0-15 cm)

\begin{tabular}{|c|c|c|c|c|c|}
\hline Solo(1) & Material de origem & P-Bray & Argila ${ }^{(2)}$ & Silte & Areia \\
\hline & & $\mathrm{mg} \mathrm{kg}^{-1}$ & $\longrightarrow$ & $\mathrm{kg}^{-1}$ & - \\
\hline 1. Typic Argiudoll Colonia Palma & Sedimentos quaternários areno-argilosos & 12 & 302 & 248 & 450 \\
\hline 2. Typic Hapludert I tapebí & Sedimentos argilo-siltosos (intemperização do basalto) & 8 & 460 & 420 & 120 \\
\hline 3. Typic Argiudoll Algorta & Arenito do Cretáceo & 14 & 100 & 99 & 801 \\
\hline 4. Typic Argiudoll Bequeló & Loess do Mioceno & 60 & 409 & 423 & 168 \\
\hline $\begin{array}{l}\text { 5. Albic Natraqualf La } \\
\text { Charqueada }\end{array}$ & Sedimentos quaternários silto-argilosos & 22 & 259 & 547 & 194 \\
\hline 6. Typic Argiudoll Alférez & $\begin{array}{l}\text { Sedimentos silto-arenosos (intemperização } \\
\text { embasamento cristalino) }\end{array}$ & 5 & 179 & 430 & 391 \\
\hline 7. Typic Argiudoll Arroyo Blanco & Siltitos Permiano & 7 & 219 & 309 & 472 \\
\hline $\begin{array}{l}\text { 8. Typic Argiudoll S.Gabriel- } \\
\text { Guaycurú }\end{array}$ & $\begin{array}{l}\text { Sedimentos silto-arenosos (intemperização do } \\
\text { embasamento cristalino) }\end{array}$ & 7 & 203 & 324 & 473 \\
\hline 9. Typic Hapludert Kiyú & Sedimentos Quaternários silto-argilosos & 11 & 453 & 465 & 82 \\
\hline 10. Typic Albaquoll Kiyú & Siltitos do quaternário & 15 & 308 & 561 & 131 \\
\hline
\end{tabular}

Quadro 2. Características químicas dos solos estudados (camada de 0-15 cm de profundidade)

\begin{tabular}{|c|c|c|c|c|c|c|c|c|c|}
\hline \multirow{2}{*}{ Solo } & \multirow{2}{*}{ 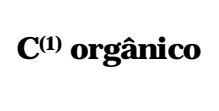 } & \multirow{2}{*}{ pH $\mathrm{H}_{2} \mathrm{O}$} & \multirow{2}{*}{ pH KCl } & \multicolumn{4}{|c|}{ Cátions trocáveis } & \multirow{2}{*}{$\mathbf{T}$} & \multirow{2}{*}{$\mathbf{v}$} \\
\hline & & & & $\mathbf{K}$ & $\mathbf{C a}$ & Mg & $\mathrm{Na}$ & & \\
\hline \multicolumn{3}{|c|}{$\mathrm{g} \mathrm{kg}^{-1}$} & & \multicolumn{5}{|c|}{$\mathrm{mmol}_{\mathrm{c}} \mathrm{kg}^{-1}$} & $\%$ \\
\hline $\begin{array}{r}1 \\
2 \\
3 \\
4 \\
5 \\
6 \\
7 \\
8 \\
9 \\
10\end{array}$ & $\begin{array}{r}24 \\
34 \\
7 \\
39 \\
17 \\
18 \\
24 \\
21 \\
39 \\
18\end{array}$ & $\begin{array}{l}5,2 \\
5,7 \\
5,2 \\
5,5 \\
5,4 \\
5,4 \\
5,4 \\
5,1 \\
6,2 \\
5,4\end{array}$ & $\begin{array}{l}4,1 \\
4,5 \\
4,1 \\
4,6 \\
4,2 \\
4,3 \\
4,3 \\
4,1 \\
5,3 \\
4,2\end{array}$ & $\begin{array}{l}0,2 \\
0,4 \\
0,1 \\
1,4 \\
0,2 \\
0,2 \\
0,3 \\
0,4 \\
0,7 \\
0,4\end{array}$ & $\begin{array}{r}13 \\
24 \\
2 \\
28 \\
6 \\
6 \\
12 \\
4 \\
30 \\
8\end{array}$ & $\begin{array}{l}3 \\
6 \\
1 \\
3 \\
2 \\
2 \\
2 \\
1 \\
5 \\
2\end{array}$ & $\begin{array}{l}0,2 \\
0,1 \\
0,2 \\
0,3 \\
0,4 \\
0,3 \\
0,2 \\
0,4 \\
0,8 \\
0,5\end{array}$ & $\begin{array}{r}19 \\
32 \\
4 \\
32 \\
12 \\
11 \\
16 \\
11 \\
38 \\
15\end{array}$ & $\begin{array}{l}86 \\
95 \\
85 \\
99 \\
78 \\
79 \\
90 \\
53 \\
96 \\
66\end{array}$ \\
\hline
\end{tabular}

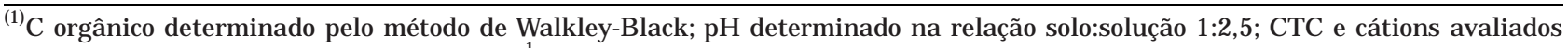
pelo método por acetato de amônio $1 \mathrm{~mol} \mathrm{~L}^{-1} \mathrm{a} \mathrm{pH} 7$.

de centrífuga de $50 \mathrm{ml}$, junto com 1,25 g de ditionito de sódio e $25 \mathrm{ml}$ de água deionizada, e agitadas durante $16 \mathrm{~h}$. A suspensão foi diluída até $60 \mathrm{ml}$ com água destilada, ajustando o pH na faixa de 3,5-4,0, e, após filtrada e diluída até $100 \mathrm{ml}$, foi determinado o conteúdo de ferro; b) ferro extraído pel o oxalato de amônio 0,2 mol L-1 a pH $3\left(\mathrm{Fe}_{03}\right)$, avaliado pela técnica ajustada por Schwertmann (1973) e descrita em Tedesco et al. (1985), pela qual as amostras dos solos foram agitadas na ausência de luz com oxalato de amônio 0,2 mol L-1, por duas horas, após as quais as suspensões foram centrifugadas, determinado-se oteor ferrona solução; c) ferro extraído pel o oxalato de amônio $0,2 \mathrm{~mol} \mathrm{~L}^{-1}$ a pH 6 ( $\left.\mathrm{Fe}_{06}\right)$ : esse método difere do anterior somente no pH da solução extratora; d) ferro extraído, seqüencialmente, por uma sol ução de $\mathrm{HCl} 8 \mathrm{~mol}$ L-1, avaliado pela técnica proposta por Segalen e descrita por Cayssials \& Puentes (1974). Na mesma amostra de solo, foi realizada uma seqüência de oito extrações, de $30 \mathrm{~s}$ cada, com HCl $8 \mathrm{~mol} \mathrm{L-1}$, centrifugando-se e determinando-se o teor de ferro em cada extrato. Posteriormente, foram plotados em um gráfico os dados de extração acumulada de ferro em função do 
número de extração. Com os valores acumulados correspondentes às extrações três a oito, ajustou-se uma reta. A extrapolação da reta corta o eixo das ordenadas $(Y)$ num ponto onde o valor de ferro extraído corresponde às formas de menor cristalinidade $\left(\mathrm{Fe}_{\mathrm{mc}}\right)$. O ferro total extraído nas oito extrações é equivalente ao ferro total presente em óxidos $\left(\mathrm{Fe}_{\mathrm{tc}}\right)$.

\section{Avaliação da adsorção de fósforo}

Em tubos de centrífuga de $50 \mathrm{ml}$, equilibraramse duplicatas de $2,5 \mathrm{~g}$ de solo com $25 \mathrm{ml}$ de uma solução de $\mathrm{CaCl}_{2}$ 0,01 mol L-1, com concentrações variáveis de fósforo adicionado como $\mathrm{Ca}\left(\mathrm{H}_{2} \mathrm{PO}_{4}\right)_{2}$. As concentrações de fósforo nas soluções foram de 0,5 , $10,15,25,40,60$ e $100 \mathrm{mg} \mathrm{L}-1$, equival entes a adições de $0,50,100,150,250,400,600$ e 1.000 mg de $P$ kg-1 $^{-1}$ de solo, respectivamente. Para inibir a flora microbiana, foram adicionadas duas gotas de tolueno. As amostras dos solos foram equilibradas com as soluções, durante $18 \mathrm{~h}$ a $25^{\circ} \mathrm{C}$ num agitador recíproco (50 ciclos por minuto), centrifugadas e filtradas. O teor de fósforo na solução foi determinado pelo método da redução sel etiva com cloreto de estanho, depois da adição de molibdato de amônio. O fósforo adsorvido pela fase sól ida foi cal culado por diferença entre o fósforo adicionado e o quantificado na solução de equilíbrio, depois de prévia correção do fósforo inicialmente presente na solução. Os valores obtidos para cada solo foram ajustados à equação de Langmuir de acordo com:

$$
\mathrm{x} / \mathrm{m}=\mathrm{K}_{1} \mathrm{~K}_{2} \mathrm{C} /\left(1+\mathrm{K}_{1} \mathrm{C}\right)
$$

em que

$\mathrm{x} / \mathrm{m}=$ quantidade de $\mathrm{P}$ adsorvido por unidade de peso de solo;
$\mathrm{K}_{1}=$ constante relacionada com energia de ligação;

$\mathrm{K}_{2}$ =adsorção máxima;

$\mathrm{C}=$ concentração de equilíbrio de $\mathrm{P}$.

Da linearização da equação de Langmuir $\left[\mathrm{C} / \mathrm{x} / \mathrm{m}=\left(1 / \mathrm{K}_{1} \mathrm{~K}_{2}\right)+\mathrm{C} / \mathrm{K}_{2}\right]$, calcularam-se $\mathrm{K}_{2}$ (gradiente-1) e $\mathrm{K}_{1}$ (gradiente/intercepto).

Osíndices $\mathrm{P}_{150}$ e $\mathrm{P}_{600}$ representama porcentagem de fósforo adsorvido pelo solo com a adição equivalente a 150 e $600 \mathrm{mg}^{\mathrm{de}} \mathrm{P} \mathrm{kg}^{-1}$ de solo.

Com os dados obtidos, real izaram-se análises de regressão e correlação. Foram adotados os procedimentos PROC REG e PROC CORR (SAS I nstitute, 1985). Para comparar os parâmetros nas equações de regressão, utilizou-se o programa MSTAT-C (Nissen, 1989).

\section{RESULTADOS E DISCUSSÃO}

As quantidades de ferro extraídas pelo ditionito $\left(\mathrm{Fe}_{\mathrm{d}}\right)$ variaram, entre os solos, de 1.598 a $8.592 \mathrm{mg} \mathrm{kg}^{-1}$ de solo, tendo sido encontrado o teor mais el evado no solo desenvolvido a partir de materiais quaternários (solo 2, Hapludert Itapebi) com influência da alteração de basal to (Quadro3). O solo que apresentou o menor teor de ferro (solo 3, Argiudoll Algorta) foi desenvolvido a partir de arenitos do Cretáceo, que revelaram baixos teores de argila (Quadro 1). Com a exceção do Hapludert Kiyu, para os demais solos, encontrou-se uma correl ação entre o ferro extraído pel o ditionito $\left(\mathrm{Fe}_{\mathrm{d}}\right)$ e o teor de argila dos solos (Figura 1), o que indica que a alta proporção dos óxidos de ferro nesses sol os encontra-se associada às frações finas.

\section{Quadro 3. Teores totais, frações e valores relativos de óxidos de ferro em dez solos do Uruguai}

\begin{tabular}{|c|c|c|c|c|c|c|c|c|c|c|c|}
\hline Solo & $F e_{d}^{(1)}$ & $F e_{03}{ }^{(2)}$ & $\mathrm{Fe}_{06}{ }^{(3)}$ & $F e_{t c}^{(4)}$ & $F e_{m c}{ }^{(5)}$ & $\frac{F e_{03}}{F e_{d}}$ & $\frac{F e_{06}}{F e_{d}}$ & $\begin{array}{l}F e_{06} \\
F e_{03}\end{array}$ & $\frac{F \mathbf{e}_{\mathrm{d}}}{\mathrm{Fe}_{\mathrm{tc}}}$ & $\frac{F e_{m c}}{F e_{t c}}$ & $\begin{array}{l}\mathrm{Fe}_{\mathrm{o3}} \\
\mathrm{Fe}_{\mathrm{mc}}\end{array}$ \\
\hline & - & $\ldots$ & $-\mathrm{mg} \mathrm{kg}^{-1}$ & 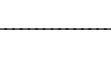 & 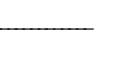 & - & - & 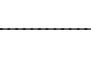 & 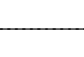 & 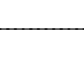 & - \\
\hline $\begin{array}{r}1 \\
2 \\
3 \\
4 \\
5 \\
6 \\
7 \\
8 \\
9 \\
10\end{array}$ & $\begin{array}{l}6.220 \\
8.592 \\
1.598 \\
4.070 \\
2.955 \\
4.490 \\
2.957 \\
4.060 \\
1.887 \\
5.653\end{array}$ & $\begin{array}{r}4.013 \\
6.235 \\
837 \\
1.838 \\
1.732 \\
2.908 \\
2.308 \\
3.175 \\
1.075 \\
3.633\end{array}$ & $\begin{array}{r}508 \\
1.642 \\
242 \\
424 \\
792 \\
508 \\
417 \\
855 \\
81 \\
758\end{array}$ & $\begin{array}{l}7.021 \\
8.879 \\
2.553 \\
3.078 \\
2.374 \\
4.877 \\
3.366 \\
4.717 \\
2.730 \\
5.643\end{array}$ & $\begin{array}{l}5.932 \\
7.592 \\
2.147 \\
1.918 \\
1.737 \\
3.552 \\
2.514 \\
3.663 \\
1.383 \\
3.993\end{array}$ & $\begin{array}{l}64 \\
73 \\
52 \\
45 \\
59 \\
65 \\
78 \\
78 \\
57 \\
64\end{array}$ & $\begin{array}{r}8 \\
19 \\
15 \\
10 \\
27 \\
11 \\
14 \\
21 \\
4 \\
13\end{array}$ & $\begin{array}{l}13 \\
26 \\
28 \\
23 \\
46 \\
18 \\
18 \\
26 \\
8 \\
21\end{array}$ & $\begin{array}{r}89 \\
97 \\
63 \\
132 \\
124 \\
92 \\
88 \\
86 \\
69 \\
100\end{array}$ & $\begin{array}{l}84 \\
86 \\
84 \\
62 \\
73 \\
73 \\
75 \\
78 \\
51 \\
71\end{array}$ & $\begin{array}{r}68 \\
82 \\
39 \\
968 \\
100 \\
82 \\
92 \\
87 \\
787 \\
91\end{array}$ \\
\hline Média & 4.248 & 2.775 & 623 & 4.524 & 3.443 & 64 & 14 & 23 & 94 & 74 & 81 \\
\hline
\end{tabular}

${ }^{(1)} \mathrm{Fe}_{\mathrm{d}}=$ extração com ditionito; ${ }^{(2)} \mathrm{Fe}_{03}=$ extração com oxalato de amônio 0,2 mol L-1 a pH 3. ${ }^{(3)} \mathrm{Fe}_{06}=$ extração com oxalato de amônio $0,2 \mathrm{~mol} \mathrm{~L}^{-1}$ a pH 6. ${ }^{(4)} \mathrm{Fe}_{\mathrm{tc}}=$ total da extração seqüencial com HCl $8 \mathrm{~mol} \mathrm{~L}^{-1} ;{ }^{(5)} \mathrm{Fe}_{\mathrm{mc}}=$ calculado por extrapolação da curva de extração seqüencial. 


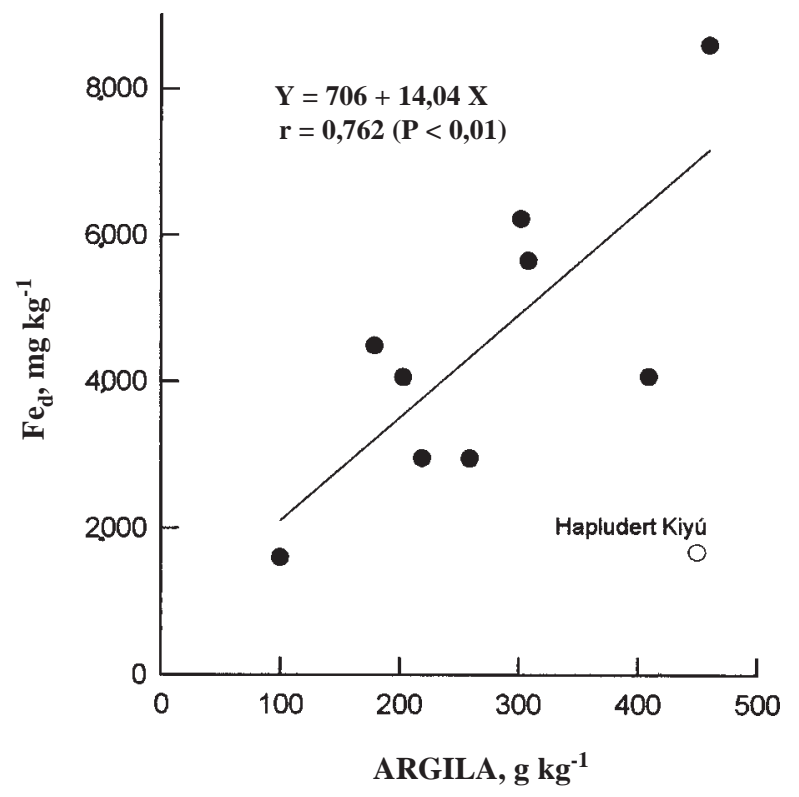

Figura 1. Relação entre a fração de ferro extraída por ditionito de sódio $\left(\mathrm{Fe}_{\mathrm{d}}\right)$ e o teor de argila dos solos.

As quantidades de ferro extraídas pelo oxalato de amônio a pH $3\left(\mathrm{Fe}_{03}\right)$ variaram de 837 a $6.235 \mathrm{mg} \mathrm{kg}^{-1}$, 45 a $78 \%$ das quantidades extraídas pel o ditionito, ficando a média para os dez solos em $64 \%$ (Quadro 3). I sto indica que, na maioria desses solos, mais da metade dos óxidos de ferro estão sob formas de baixa cristalinidade. As condições de formação dos solos, com períodos alternados de oxidação-redução, constituem causas prováveis dessa alta proporção (Munch et al., 1978; Blume, 1988). O oxalato de amônio a pH 6 ( $\left.\mathrm{Fe}_{06}\right)$ extraiu menores quantidades de ferro que o oxalato a $\mathrm{pH} 3$ (Quadro 3), correspondendo, na média, a 23\% da fração extraída pelo $\mathrm{Fe}_{03}$. O Natraqualf $\mathrm{La}$ Charqueada apresentou a maior relação $\mathrm{Fe}_{06} / \mathrm{Fe}_{03}$ (46\%) entre os sol os estudados; trata-se de um solo que ocupa posições baixas e planas da paisagem, com um horizonte subsuperficial argiloso muito desenvolvido, que afeta a drenagem interna; é um dos principais solos cultivados com arroz no país.

As quantidades de ferro extraídas seqüencialmente pelo $\mathrm{HCl} 8 \mathrm{~mol} \mathrm{~L}^{-1}\left(\mathrm{Fe}_{\mathrm{tc}}\right)$ foram, na média, cerca de $6 \%$ superiores às extraí das pelo ditionito; entretanto, em três solos, foram inferiores. Similarmente, as quantidades correspondentes às formas de menor cristalinidade $\left(\mathrm{Fe}_{\mathrm{mc}}\right)$, cal culadas a partir da extração seqüencial pela extrapolação da curva ao eixo das ordenadas, foram superiores (relação $\mathrm{Fe}_{03}$ / $\mathrm{Fe}_{\mathrm{mc}}=81 \%$ ) às extraídas pelo oxalato de amônio a pH 3 (Quadro 3).

Na figura 2, são apresentadas as isotermas de adsorção de fósforo correspondentes a cinco dos dez sol os estudados. Observou-se a relação direta entre as quantidades de fósforo adsorvidas e as quantidades de óxidos de ferro presentes: o Hapludert Itapebi, com maiores teores, mostrou máxima adsorção de fósforo, enquanto o Argiudoll Algorta, com menores teores, revelou menor adsorção. As curvas de adsor ção dos cinco solos não apresentadas na figura 2 são semelhantes ou intermediárias às curvas de adsorção plotadas.

Os índices de adsorção de fósforo para os dez sol os estão no quadro 4. A capacidademáxima de adsor ção de fósforo $\left(1 / K_{2}\right)$ variou, entre solos, de 104 a $704 \mathrm{mg} \mathrm{kg}^{-1}$. Os valores para os índices $\mathrm{P}_{150}$ situaram-se entre 42 e $98 \%$, ao passo que o índice para o $\mathrm{P}_{600}$ ficou entre 14 e $80 \%$. Altos teores de sesquióxidos de ferro são, muitas vezes, considerados como o fator determinante da adsorção do fósforo em solos (Uehara \& Gillman, 1981). As formas de baixa cristalinidade devem fixar mais fósforo que as cristal inas. Neste trabal ho, encontraram-sebaixas correlações (Quadro 5) entre os índices de adsorção de fósforo e as formas cristalizadas de sesquióxidos de ferro. I sto é, a diferença $\mathrm{Fe}_{d}-\mathrm{Fe}_{03}$ que é considerada boa estimativa das formas cristalinas de ferro (Smith \& Mitchell, 1987; Delvaux et al., 1989) ea diferença $\mathrm{Fe}_{\mathrm{tc}}-\mathrm{Fe}_{\mathrm{mc}}$ quetambém indicaria as formas cristalinas, apresentaram baixos coeficientes de correlação com os índices de adsorção de fósforo (Quadro 5). O índice de adsorção máxima de fósforo $\left(\mathrm{K}_{2}\right)$ correlacionou-se significativamente ( $r=0,894, P<0,01)$ com a quantidade de óxidos de ferro extraída pelo oxalato de amônio $0,2 \mathrm{~mol} \mathrm{L-1}$ $\left(\mathrm{Fe}_{03}\right)$ a pH 3 (Figura 3).

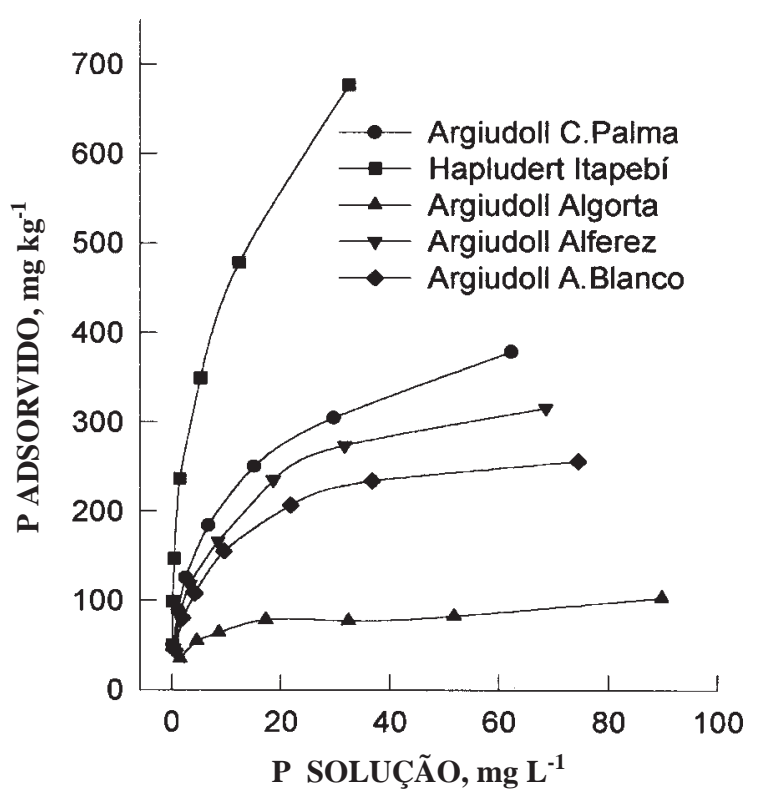

Figura 2. I sotermas de adsorção em cinco solos selecionados. 


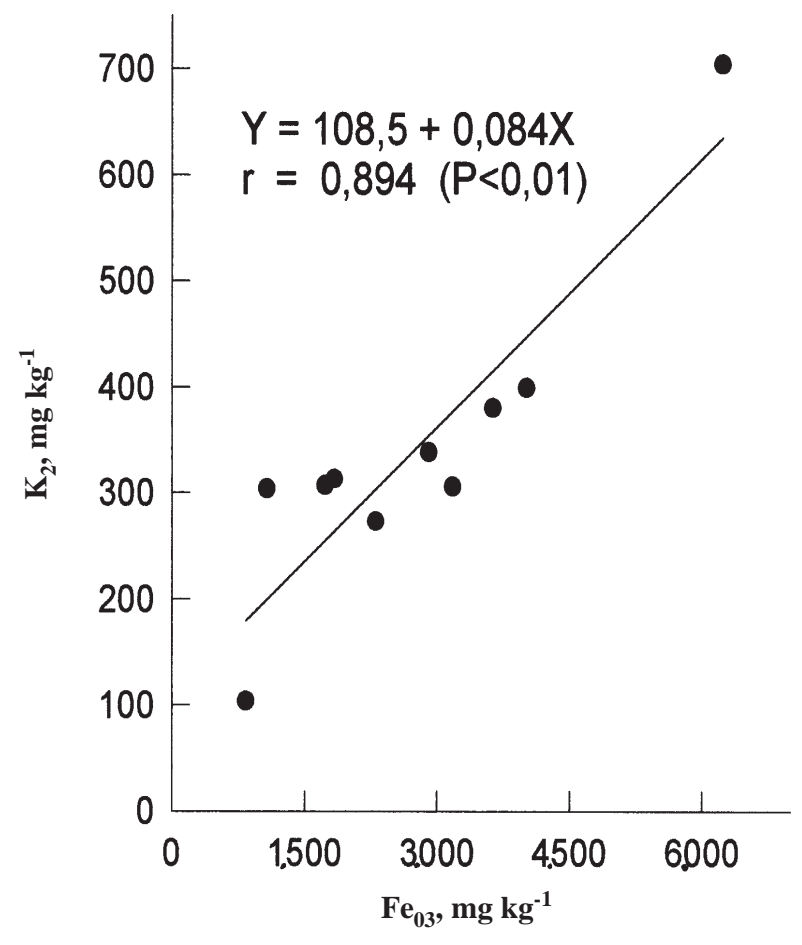

Figura 3. Relação entre o teor de óxido de ferro extraído pelo oxalato de amônio 0,2 mol L-1 pH 3 e a capaci dade máxi ma de adsorção de fósforo dos solos.

Outros autores encontraram altas correlações entre a adsorção de fósforo e o ferro extraído pelo oxalato de amônio a pH 3 (Bromfield, 1965; Sree Ramulu et al., 1967; Lewis et al., 1981; M olina et al., 1991; Soon, 1991). U ma comprovação da alta capacidade dessa fração dos óxi dos de ferro de reagir com o fósforo foi obtida por Syers et al. (1971), que encontraram, após remoção da fração dos óxidos de ferro pelo oxalato de amônio a pH 3, redução da adsorção de fósforo em amostras de solos do Rio Grande do Sul.

O índice $\mathrm{P}_{600}$ correlacionou-se significativamente ( $r=0,975, p<0,01)$ com a capacidade máxima de adsorção dos sol os (Quadro 5) e ( $r=0,894, P<0,01$ ) com as formas deferro $\left(\mathrm{Fe}_{03}\right)$ de baixa cristalinidade (Figura 4), podendo ser, também, utilizado como um índice para estimar a capacidade de adsorção de fósforo desses solos.

As rel ações dos índices de adsor ção de fósforo com $\mathrm{Fe}_{\mathrm{mc}}$, embora significativas, apresentaram coeficientes de correlação mais baixos (Quadro 5).

A constante $K_{1}$, relacionada com a energia de adsorção, mostrou um coeficiente el evado para $\mathrm{Fe}_{06}$, o qual apresentou menor correl ação com o parâmetro $\mathrm{K}_{2}$ do que o $\mathrm{Fe}_{03}$, e uma correlação baixa com o $\mathrm{P}_{600}$ $(r=0,678, P<0,01)$. SegundoWillett \& Cunningham
Quadro 4. Coeficientes da equação linearizada de Langmuir ( $a=$ intercepção e $\mathbf{b}=$ declividade), coeficientes de correlação ( $r$ ), adsorção máxima $\left(K_{2}\right)$, energia de ligação $\left(K_{1}\right)$ e porcentagem de fósforo adsorvido pelos solos com a adição de $150 \mathrm{mg} \mathrm{kg}^{-1}$ de $P$ de solo ( $P_{150}$ ) e de $600 \mathrm{mg} \mathrm{kg}^{-1}$ de $P$ de solo ( $\left.P_{600}\right)$

\begin{tabular}{rlllllll}
\hline & \multicolumn{1}{c}{$\mathbf{a}$} & $\mathbf{b}$ & & & & & \\
Solo & $\mathbf{x} \mathbf{1 0}^{-\mathbf{2}}$ & $\mathbf{x} \mathbf{1 0}^{-\mathbf{3}}$ & $\mathbf{r}$ & $\mathbf{K}_{\mathbf{2}}$ & $\mathbf{K}_{\mathbf{1}}$ & $\mathbf{P}_{\mathbf{1 5 0}}$ & $\mathbf{P}_{\mathbf{6 0 0}}$ \\
& & & & & & & $-\%-$ \\
& & & & & & & - \\
1 & 1,46 & 2,5 & $0,992^{* *}$ & 400 & 0,170 & 84 & 51 \\
2 & 0,4 & 1,42 & $0,986 * *$ & 704 & 0,355 & 98 & 80 \\
3 & 5,93 & 9,64 & $0,090^{* *}$ & 104 & 0,162 & 43 & 14 \\
4 & 2,98 & 3,19 & $0,992^{*}$ & 313 & 0,107 & 73 & 42 \\
5 & 1,62 & 3,26 & $0,996 * *$ & 307 & 0,201 & 79 & 43 \\
6 & 1,77 & 2,97 & $0,996 * *$ & 334 & 0,168 & 79 & 47 \\
7 & 2,1 & 3,66 & $0,998^{* *}$ & 273 & 0,174 & 72 & 39 \\
8 & 1,45 & 3,27 & $0,996 * *$ & 306 & 0,226 & 83 & 44 \\
9 & 3,09 & 3,29 & $0,992^{* *}$ & 304 & 0,106 & 69 & 39 \\
10 & 1,25 & 2,63 & $0,996 * *$ & 380 & 0,210 & 86 & 53 \\
\hline
\end{tabular}

** significativo ao nível de $1 \%$.

Quadro 5. Coeficientes de correlação $(r)$ e suas probabilidades de significância, entre a capacidade máxi ma de adsorção de fósforo $\left(K_{2}\right)$, energia de ligação $\left(K_{1}\right)$, índices $P_{150}$ e $P_{600}$ e formas de ferro nos dez solos estudados

\begin{tabular}{cllll}
\hline Forma de ferro & $\mathbf{K}_{\mathbf{2}}$ & $\mathbf{K}_{\mathbf{1}}$ & $\mathbf{P}_{\mathbf{1 5 0}}$ & $\mathbf{P}_{\mathbf{6 0 0}}$ \\
\hline $\mathrm{K}_{1}$ & 0,714 & - & - & - \\
& 0,01 & - & - & - \\
$\mathrm{P}_{150}$ & 0,848 & 0,557 & - & - \\
& 0,01 & 0,05 & - & - \\
$\mathrm{P}_{600}$ & 0,975 & 0,678 & - & - \\
& 0,01 & 0,02 & - & - \\
$\mathrm{Fe}_{03}{ }^{(1)}$ & 0,894 & 0,800 & 0,812 & 0,894 \\
$\mathrm{Fe}_{06}{ }^{(2)}$ & 0,01 & 0,01 & 0,01 & 0,01 \\
$\mathrm{Fe}_{\mathrm{mc}}{ }^{(3)}$ & 0,806 & 0,949 & 0,714 & 0,800 \\
$\mathrm{Fe}_{\mathrm{d}}-\mathrm{Fe}_{03}$ & 0,01 & 0,01 & 0,01 & 0,01 \\
$\mathrm{Fe}_{\mathrm{tc}}-\mathrm{Fe}_{\mathrm{mc}}{ }^{(4)}$ & 0,800 & 0,748 & 0,640 & 0,762 \\
& 0,01 & 0,01 & 0,03 & 0,01 \\
& 0,648 & 0,100 & 0,557 & 0,640 \\
& 0,02 & 0,32 & 0,06 & 0,03 \\
& 0,469 & 0,332 & 0,574 & 0,557 \\
\end{tabular}

(1) extração com oxalato de amônio $0,2 \mathrm{~mol} \mathrm{~L}^{-1}$ a pH 3. (2) extração com oxalato de amônio $0,2 \mathrm{~mol} \mathrm{~L}^{-1}$ a pH 6. (3) calculado a partir de $\mathrm{Fe}_{\mathrm{tc}}$; intercepção da reta de regressão com os eixos das ordenadas (vide texto). ${ }^{(4)} \mathrm{Fe}_{\mathrm{tc}}=$ total da extração seqüencial com $\mathrm{HCl} 8 \mathrm{~mol} \mathrm{~L}^{-1}$. 


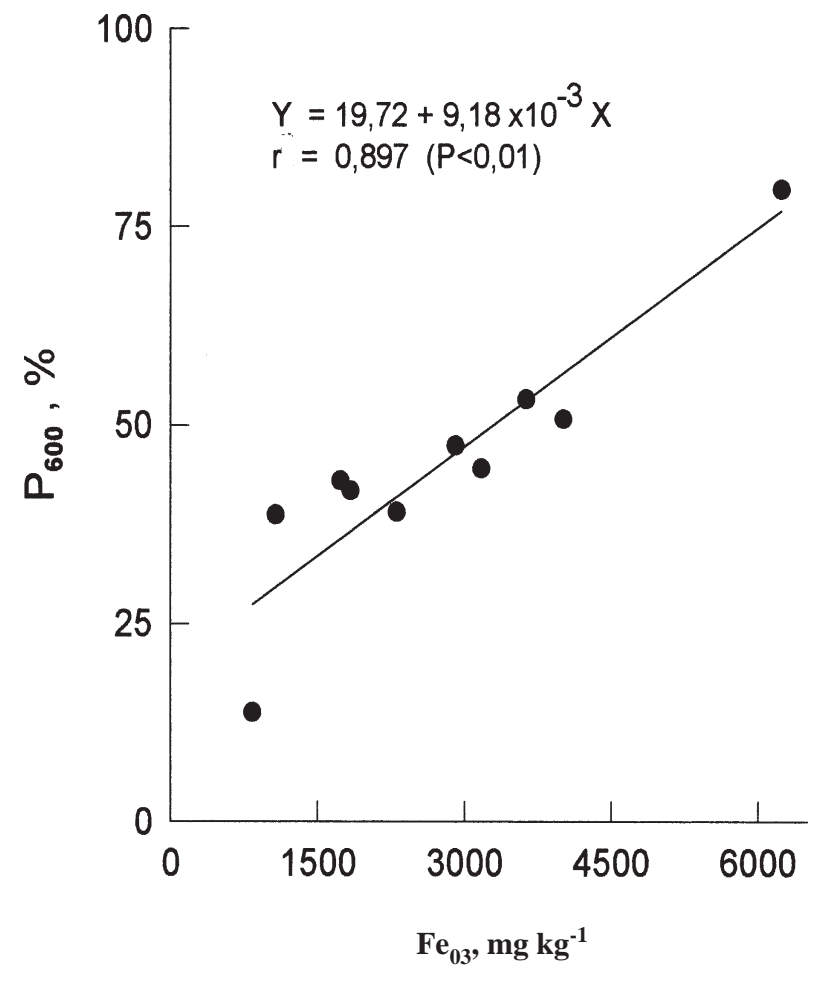

Figura 4. Relação entre os óxidos de ferro extraídos pelo oxalato de amônio 0,2 mol L-1 a pH 3 e a porcentagem de fósforo adsorvida pelos solos com a adição de uma dose de fósforo equivalente a $600 \mathrm{mg} \mathrm{kg}^{-1}$ de solo.

(1983), o significado físico-químico dessa constante não é muito claro, como o parâmetro que estima a adsorção máxima do fósforo $\left(\mathrm{K}_{2}\right)$.

\section{CONCLUSÕES}

1. Os teores de óxidos de ferro extraídos pelo ditionito $\left(\mathrm{Fe}_{\mathrm{d}}\right)$ variaram de 1.598 a $8.592 \mathrm{mg} \mathrm{kg}^{-1}$, sendo diretamenterelacionados com oteor de argila dos solos, determinados pel o material de origem.

2. A capacidade máxima de adsorção de fósforo variou de 104 a $704 \mathrm{mg} \mathrm{kg}^{-1}$ de sol o e correl acionouse significativamente com as formas de óxidos de ferro de baixa cristalinidade, que representaram de 45 a $78 \%$ do total extraído pelo ditionito $\left(\mathrm{Fe}_{03} / \mathrm{F}_{\mathrm{d}}\right)$.

3. A porcentagem de fósforo adsorvida após a adição de $600 \mathrm{mg}$ de $\mathrm{P} \mathrm{kg} \mathrm{kg}^{-1}$ de solo $\left(\mathrm{P}_{600}\right)$ apresentou alta correlação com a capacidade máxima de adsorção de fósforo dos sol os e com as formas de ferro de baixa cristalinidade. Tal índice pode ser utilizado para estimar a adsorção de fósforo nesses solos.

\section{LITERATURA CITADA}

BALLARD, R.\& FISKELL, J.G.A. Phosphorus reaction in Coastal Plain Forest soils: I. Relationship to soil properties. Soil Sci. Soc. Am. Proc., 38:250-255, 1974.

BLUME, H.P. The fate of iron during soil formation in humidtemperate environments. In: STUCKI, J.W., ed. Iron in soils and clay minerals. Holland, D. Reidel Publishing, 1988. p.749-777.

BRADLEY, J .; VIMPANY, I.\& NICHOLLS, P.J . Effects of water logging and subsequent drainage of a pasture soil on phosphate sorption, extractable phosphate and oxalateextractable iron. Aust. J. Soil Res., 22:455-461, 1984.

BROMFIELD, I.S.M. Studies of the relative importance of iron and aluminum in the sorption of phosphate by some australian soils. Aust. J. Soil Res., 3:31-44, 1965.

CAYSSIALS, R. \& PUENTES, R. Determinación y fraccionamiento de hierroy aluminiolibreen algunos suel os del U ruguay. Montevideo, Facultad deAgronomía, 1974. 88p. (Tesis Ingeniero Agrónomo)

DELVAUX, B.; HERBILLON, A.J. \& VIELVOYE, L. Characterization of a weathering sequence of soils derived from volcanic ash in Cameroon. Taxonomic, mineralogical and agronomic implications. Geoderma, 45:375-388, 1989.

DICK, D.P. Caracterizaçao de óxi dos de ferro eadsorção de fósforo na fração argila de horizontes B latossólicos. Porto Alegre, Universidade Federal do Rio Grande do Sul, 1986. 196p. (Tese de Mestrado)

ESTADOS UNIDOS. Department of Agriculture. Soil Survey Staff. Keys to soil taxonomy, 5. Blaksburg, Pocahontas, 1992. 56p. (SMSS Technical Monograph, 19)

HERNANDEZ, J .; OTEGUI, O.\& ZAMALVIDE, J .P. Formas y contenidos de fósforo en algunos suelos del Uruguay. Montevideo, Facultad de Agronomía, 1985. 32p. (Boletín de Investigaciones, 43)

KÄMPF, N. O ferro no solo. In: REUNIÃO SOBRE FERRO EM SOLOS INUNDADOS, 1., Goiania, 1988. Anais. Goiânia, EMBRAPA-CNAF, 1988. p.35-71 (Documentos, 22).

KHALID, R.A.; PATRICK, W.H.\& DE LAUNE, R.D. Phosphorus sorption characteristics of flooded soils. Soil Sci. Soc. Am. J ., 41:305-310, 1977.

KILMER, V. The estimation of free iron oxides in soils. Soil Sci. Soc. Am. Proc., 24:420-421, 1960.

LEWIS, D.L.; CLARKE, A.L.\& HALL., W.B. Factors affecting the retention of phosphorus applied as Superphosphate to the Sandy soils in South-eastern South Australia. Aust. J. Soil Res., 19:167-174, 1981.

MADRID, L.\& DE ARAMBARRI, P. Adsorption of phosphate by two iron oxides in relation to their porosity. J. Soil Sci., 36:523-530, 1985.

MEHRA, O.P. \& J ACKSON, M.L. I ron oxide removal from soils and clays by a dithionite-citrate system buffered with sodium bicarbonate. In: NATIONAL CONFERENCE CLAYS \& CLAY MINERALS, 7., Ottawa, 1960. Proceedings. Ottawa, 1960. p.317-327. 
MOLINA, E.; BORNEMISZA, N.; SANCHO, F. \& KASS, D.L. Soil aluminum and iron fractions and their relationships with $\mathrm{P}$ immobilization and other soil properties in andisols of Costa Rica and Panama. Comm. Soil Sci. Plant Anal., 22:1459-1476, 1991.

MUNCH, J.C.; HILLEBRAND, T.H. \& OTTOW, J.C.G. Transformations in the Feo/Fed ratio of pedogenic iron oxides affected by iron-reducing bacteria. Can. J. Soil Sci., 58:475-486, 1978.

NISSE N, O. MSTAT-C: Microcomputer Statistical Program. Ann Arbor, Michigan State University, 1989. p.104-106.

SAH, R.N.; MIKKELSEN, D.S. \& HAFEZ, A.A. Phosphorus behavior in flooded-drained soils. II. Iron transformation and phosphorus sorption. Soil Sci. Soc. Am. . ., 53:1723-1729, 1989.

SAS Institute, Inc. SAS User's Guide: Statistics. Cary, 1985.

SCHWERTMANN, U. Use of oxalate for Fe extraction from soils. Can. J. Soil Sci., 53:224-246, 1973.

SMITH, B.F.L. \& MITCHELL, B.D. Characterization of poorly ordered minerals by selective chemical methods. I $\mathrm{n}$ : WILSON, M.J ., ed. A handbook of determinative methods in clay mineralogy. New York, Blackie, 1987. p.275-294.

SOON, Y.K. Solubility and retention of phosphate in soils of the northwestern Canadian prairie. Can. J. Soil Sci., 71:453463, 1991.

SREE RAMULU, U.S.; PRATT, P.F. \& PAGE, A.L. Phosphorus fixation by soils in relation to extractable iron oxides and mineralogical composition. Soil Sci. Soc. Am. Proc., 31:193196, 1967.
SYERS, J.K.; EVANS, T.D.; WILLIAMS, J.D.H. \& MURDOCK, J.T. Phosphate sorption parameters of representative soils from Rio Grande doSul, Brazil. Soil Sci., 112:267-275, 1971.

TEDESCO, M.J .; VOLKWEISS, S.J . \& BOHNEN, H. Análises de solo, plantas e outros materiais. PortoAlegre, Universidade Federal do Rio Grande doSul, 1985. 188p. (UFRGS. Boletim Técnico, 5)

UEHARA G. \& GILLMAN G. The mineralogy, chemistry, and physics of tropical soils with variable charge clays. Boulder, Westview Press, 1981.

URUGUAY. Ministerio de Agricultura Y Pesca. Dirección de Suelos Y Fertilizantes. Carta de Reconocimiento de Suelos del Uruguay. Montevideo, 1976.

VAHL, L.C.; ANGHINONI, I. \& KAMPF, N. Previsão da acumulação de ferro (II) trocável no solo durante o alagamento através de análise química. In: CONGRESSO BRASILEIRO DE CIÊNCIA DO SOLO, 24., 1993. Goiânia, Resumos expandidos. Campinas, Sociedade Brasileira de Ciência do Solo, 1993. p.183-184.

WILLETT, I.R. \& CUNNINGHAM, R.B. Influence of sorbed phosphate on the stability of ferric hydrous oxide under controlled pH and Eh conditions. Aust. J. Soil Res., 21:301$308,1983$.

WILLETT, I.R. \& HIGGINS, M.L. Phosphate sorption by reduced and reoxidized ricesoils. Aust.J . Soil Res., 16:319-326, 1978.

WILLETT, I.R.; MUIRHEAD, W.A. \& HIGGINS, M.L. The effects of rice growing on soil phosphorus immobilization. Aust. Exp. Agric. Anim. Husb., 18:270-275, 1978.

WILLETT, I.R .\& HIGGINS, M.L. Phosphate sorption and extractable iron in soils during irrigated rice-upland crop rotations. Aust. Exp. Agric. Anim. Husb., 20:346-353, 1980. 